\title{
Prosthetic strap system for simplified ventral hernia repair: results of a porcine experimental model
}

\author{
G. Amato - G. Romano • A. Agrusa • \\ G. Cassata $\cdot$ G. Salamone $\cdot$ G. Gulotta
}

Received: 18 December 2009/Accepted: 5 March 2010/Published online: 24 March 2010

(C) Springer-Verlag 2010

\begin{abstract}
Introduction Aiming to achieve a simplified ventral hernia repair, a proprietary oval-shaped mesh was experimentally tested in a porcine model. The mesh is structured with a large central body and radiating straps. The friction of the straps passing through the tissues are hypothesized to be adequate to maintain the position of the mesh during tissue ingrowth, avoiding classic point fixation while ensuring a wide coverage of the abdomen.

Methods The mesh, having six radial straps, was placed using a sublay preperitoneal technique in four pigs. All straps were passed laterally through the abdominal wall and exteriorized from the skin. The straps were trimmed at the level of the skin, allowing the stumps to recoil into the subcutaneous space. The animals were euthanized at 1 and 4 months to determine the integration of the straps.

Results Macroscopically, all 24 straps were firmly incorporated within the abdominal wall. The tension-free placement of the mesh by using the straps was effective. The friction of the straps passing through the tissues was adequate to keep the mesh well orientated. No dislocation of the implants was observed. The strap system also allowed a broader coverage of the abdominal wall, far beyond the wound opening.
\end{abstract}

G. Amato $(\bowtie) \cdot$ G. Romano · A. Agrusa · G. Salamone ·

G. Gulotta

Department of General Surgery, Urgency, and Organ

Transplantation, University of Palermo, via del Vespro 129,

90100 Palermo, Italy

e-mail: giusamato1@tele2.it

G. Cassata

Experimental Zooprophylactic Institute, Via Rocco Dicillo 4, 90129 Palermo, Italy
Conclusions The described arm system of the aforementioned implant seems to be effective in eliminating point fixation of the mesh. The fixation arms seemed to have ensured that the mesh stayed orientated in all of the animals. A very wide lateral mesh placement was accomplished, assuring sufficient defect overlap when shrinkage occurs.

Keywords Ventral hernia - Prostheses and implants . Incorporation - Surgical fixation devices · Friction

\section{Introduction}

Ventral hernia repair represents one of the most frequent surgical procedures [1]. Postoperative complications such as seroma, hematoma, and wound sepsis still embody an issue in this type of surgical repair, having been reported in the literature with an incidence between 13 and 44\% [2-4]. This, along with a high recurrence rate of between 31 and $49 \%$ if a pure tissue repair is performed [5-8], indicates that there is room for improvement with the technique. Progress in surgical materials and techniques, such as the use of synthetic meshes, have demonstrated improved results with the reduction of the recurrences to less than $10 \%$ [9-12].

Nevertheless, the use of prosthetics presents its own problems [1]. To date, in ventral hernia repair (open as well laparoscopic hernia repair), the fixation of the mesh is often complex. Site-specific or small meshes have led to unacceptable failure rates, which has led to a shared concept that, in the case of incisional hernia repair, the mesh must be large enough to overlap the defect by an adequate amount $[13,14]$.

In open repair, the fixation of meshes is frequently difficult to achieve because of the remote and narrow location 
where the mesh margins should be fixed. In open and laparoscopic repair, suture or tack point fixation of the mesh is in evident contrast with the dynamics of the abdominal wall. This muscular movement against fixed points often results in tissue disruption, pain, bleeding, hematoma, or even mesh displacement $[14,15]$.

In order to resolve the issue of fixing the mesh, we have developed a proprietary mesh with a special design which is different to the classic fixation, allowing tensionfree and dynamic compliant features after implantation. The shape of the implant is comparable to conventional ones, but the presence of radiating mesh arms at its margins makes it somewhat unique. These arms are intended to pass through the abdominal wall structures, thus, stabilizing the implant thanks to the resistance exerted by its surface lying in contact with the tissues. The use of such mesh straps in surgery is not new, having been used in the last several years to repair female genital prolapse [16, 17]. Scientific reports demonstrate that the friction exerted by the polypropylene arms provides for an efficient stabilization. This feasibility study aims to evaluate whether, in a porcine model, the tension-free properties of a modified mesh through the use of a strap system are helpful in ventral hernia repair by avoiding the need for point fixation.

\section{Materials and methods}

This study was performed under the regulations of the University of Palermo and the Experimental Zooprophylactic Institute of Palermo according the ethical guidelines of both Institutions. A proprietary oval-shaped mesh consisting of a central body with six radiating mesh arms (straps) of 2-cm width and 15-cm length was developed by Insightra Medical Inc. of California (Freedom ${ }^{\mathrm{TM}}$ Ventral Hernia Repair-S size) (Fig. 1a). This newly developed mesh was designed to be employed in preperitoneal ventral hernia repair procedures. The mesh was constructed using lightweight large-porous polypropylene (Assut Europe SpA, Italy). The oval central body of the implant employed in our experimental model had dimensions $12 \times 15 \mathrm{~cm}$. The scope of the mesh arms was to tunnel them through the tissues of the abdominal wall with the purpose to hold the mesh in place by friction, without transmitting tension to the body of the implant and, importantly, avoiding suture fixation. The experimental trial was carried out in four pigs having a weight range of 30-40 kg. In each animal, after opening the abdominal wall with a skin incision of $8-10 \mathrm{~cm}$ in length, the peritoneum was separated from the layer of the posterior abdominal wall by manual blunt dissection (Fig. 1b). All four meshes were deployed in a
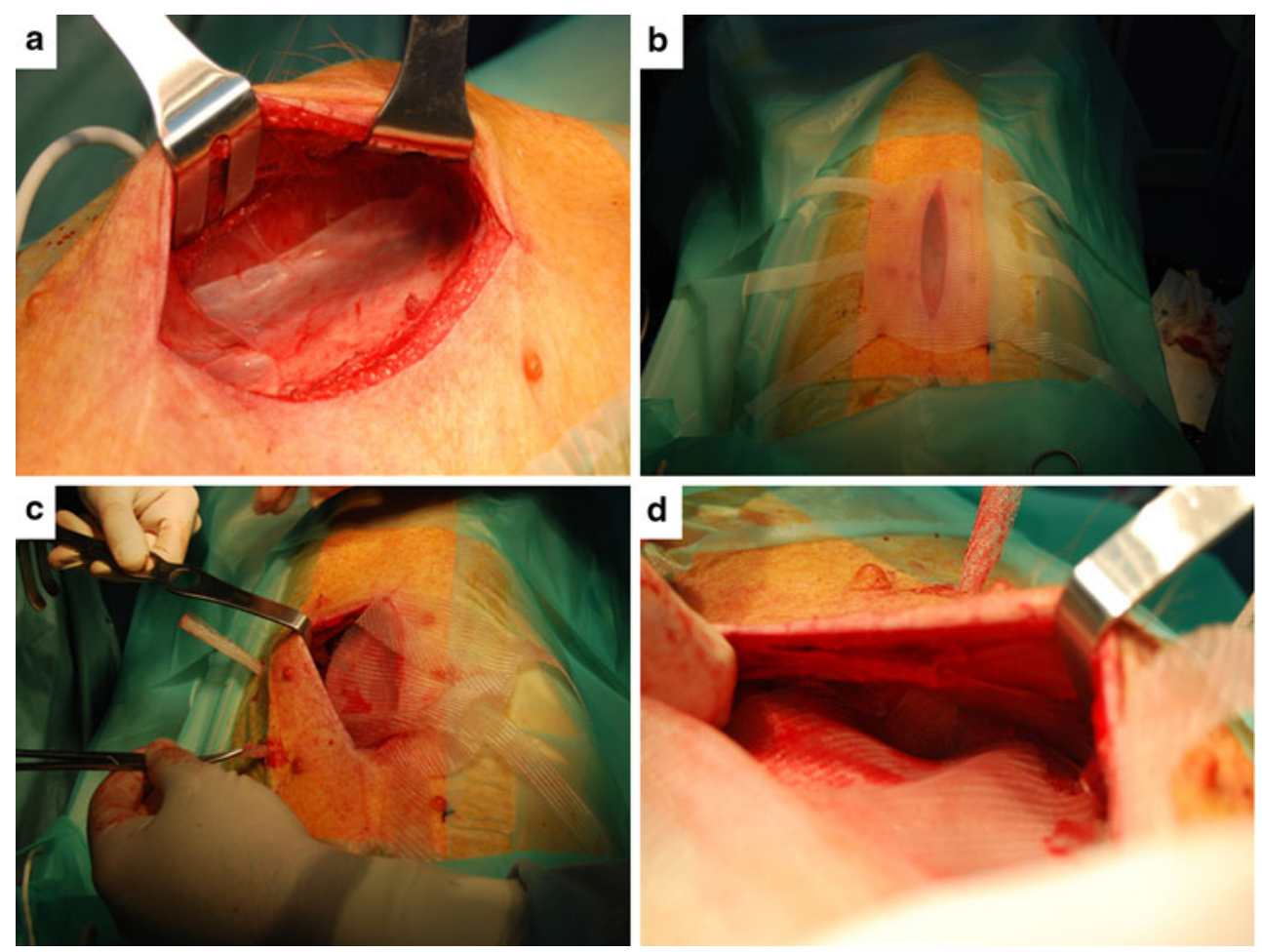

Fig. 1 The procedure. a The peritoneal sheath after manual blunt dissection from the posterior abdominal wall. b The six-arm mesh prior to delivery. $\mathbf{c}$ The arms of the mesh being introduced through the tissue tunnels. d The strap passes through the abdominal wall far away from the wound opening 

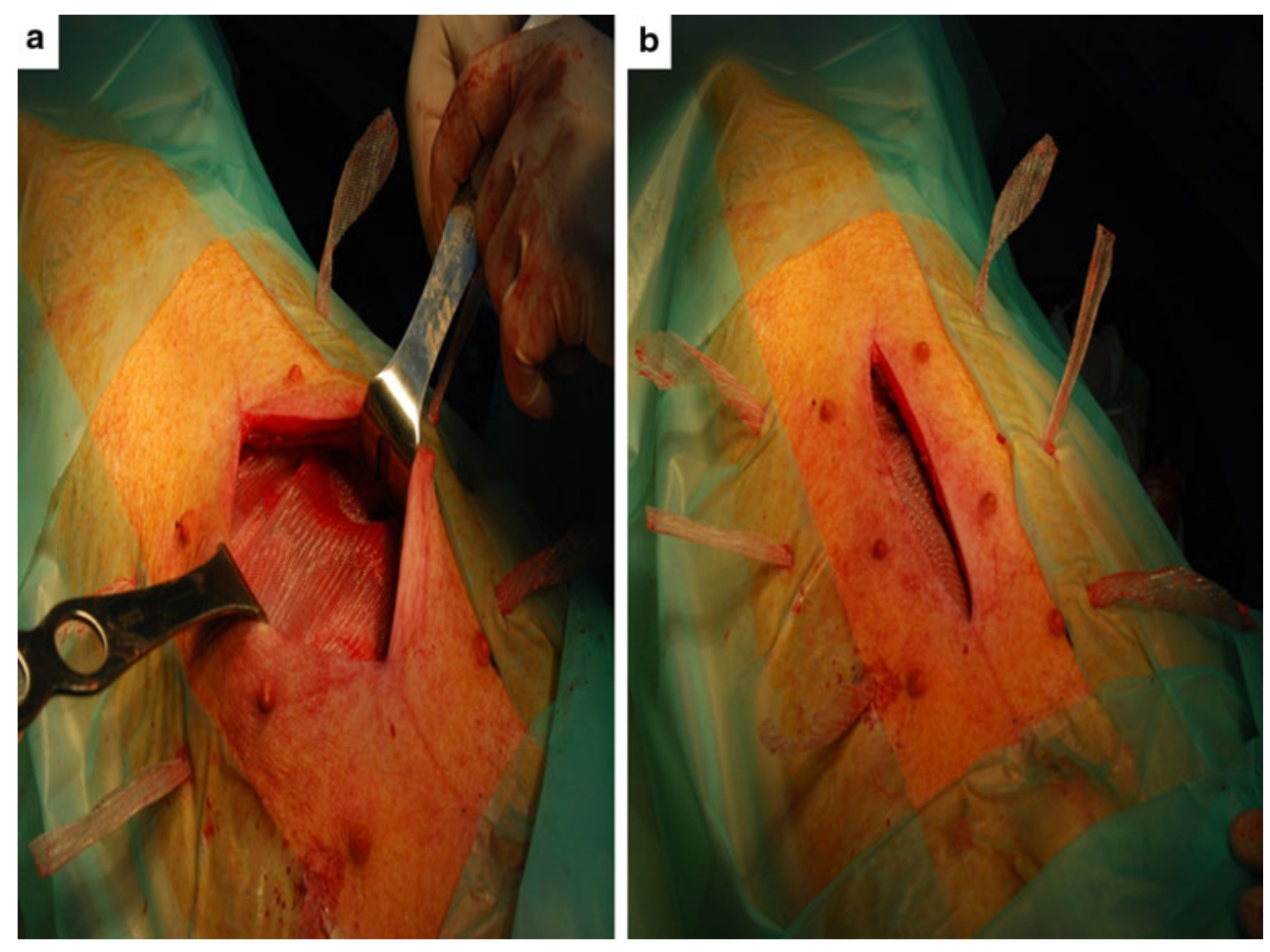

Fig. 2 a The mesh lies flat in a preperitoneal sublay placement. b All six straps are pulled out through the abdominal wall

preperitoneal sublay fashion and all straps were passed through the abdominal wall and out from the skin by means of tunneling forceps though the tissues, grasping the straps, and pulling them out (Fig. 1c, d). Of note is that the tunnel is drastically smaller than the folded mesh straps, allowing the straps to sit in a compressed form in the tunnel, adding to the gripping forces. No additional fixation was used to hold the mesh in place. All straps pulled out from the skin were trimmed in order to let the stumps retract back into the subcuticular space (Fig. 2a, b). After placement of the mesh and ensuring that it was lying flat with minimal folds, the layers of the abdominal wall were closed by suture (Fig. 3a) and the skin was closed with the total intradermal suturing technique (Fig. 3b). The small skin punctures where the straps were exteriorized were not sutured in the porcine model (Fig. 3b). The surgical procedures in the animals were carried out under general anesthesia (premedication: zolazepam + tiletamine $6.3 \mathrm{mg} / \mathrm{kg}+$ xylazine $2.3 \mathrm{mg} / \mathrm{kg}$; induction: propofol $0.5 \mathrm{mg} / \mathrm{kg}$; maintenance: isoflurane + pancuronium $0.07 \mathrm{mg} / \mathrm{kg} 15-$ $30 \mathrm{mg}$ ). All pigs received a postoperative antibiotic treatment with oxytetracycline $(20 \mathrm{mg} / \mathrm{kg} / \mathrm{die}$ for 3 days). To determine the condition of all 24 straps within the abdominal wall structures, two pigs were sacrificed at 1 month and the two after 4 months. The abdominal wall was block-dissected and reflected to examine the placement of the mesh and the position and condition of the straps. We looked macroscopically for any detrimental tissue reaction, erosions, or main-body dislocation. Histological examination of the excised straps as well as the body of the mesh was also carried out.

\section{Results}

All animals survived the operative procedures. In all pigs, the wounds healed per normal course. In the follow up, no seroma or infection was detected. At both 1 and 4 months, we could macroscopically observe that all 24 straps were completely and firmly integrated within the abdominal wall, allowing the stabilization of the mesh through the macroscopically evident ingrowth of fibrotic tissue (Fig. 4a, b). The fixation-free fastening of the mesh made by the strap was effective and avoided dislocation of the implants at a time of both 1 and 4 months from implantation. The body of the meshes appeared well orientated and tightly fastened in the abdominal wall (Fig. 5a, b). In addition, the strap system seems to have allowed the broad and effective covering of the abdominal wall, far beyond the wound opening, which is in line with the suggestions reported in the literature $[13,14]$. The histological examination showed a normal inflammatory response to polypropylene, as well as a fibroblastic incorporation of the body of the mesh and straps. 

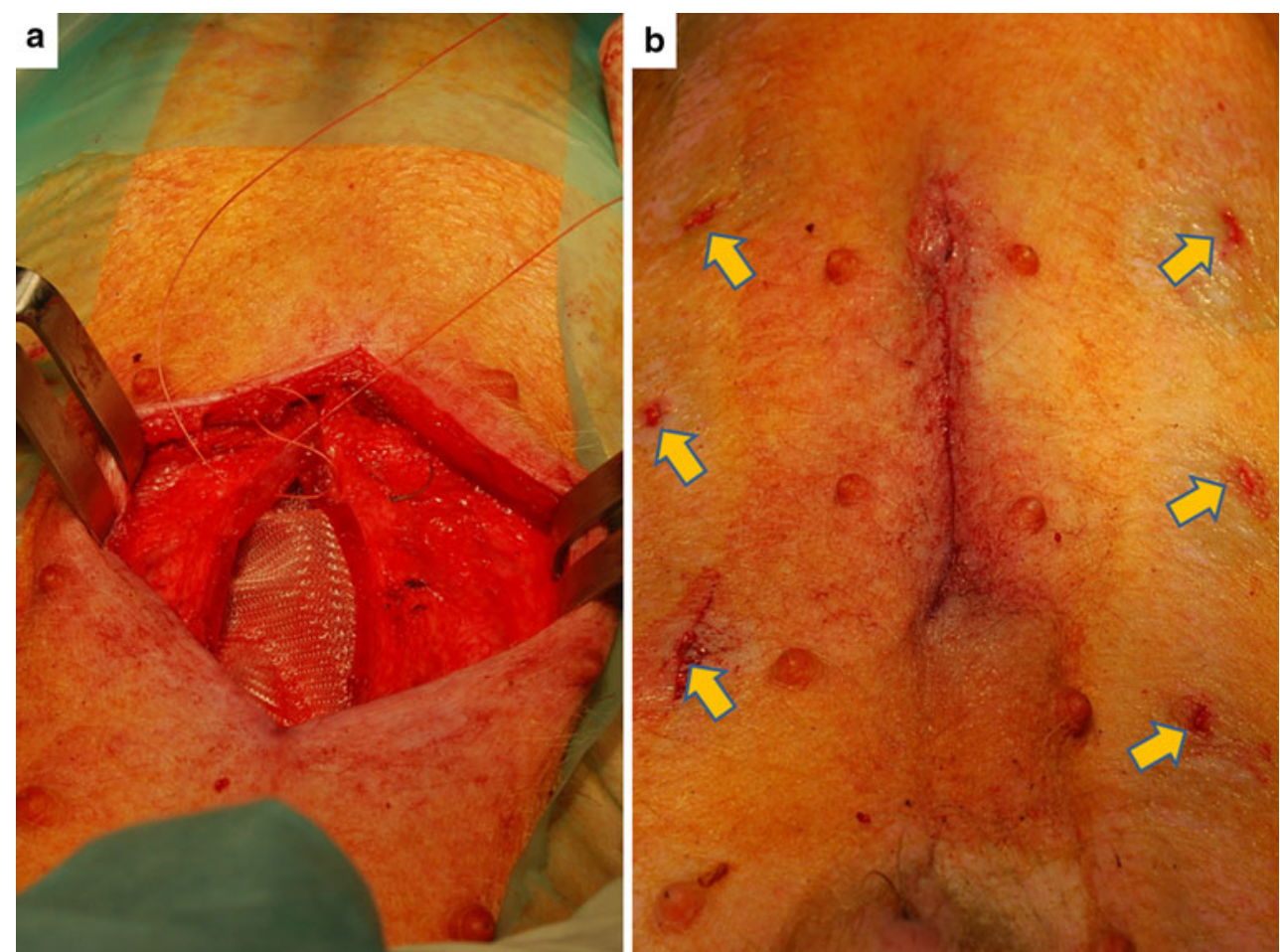

Fig. 3 a The abdominal wall layers being closed above the mesh. b Wound closed by means of total intradermal suture. Note that we did not suture the skin punctures where the arms have been pulled out (yellow arrows). The stumps sit in the subcuticular space
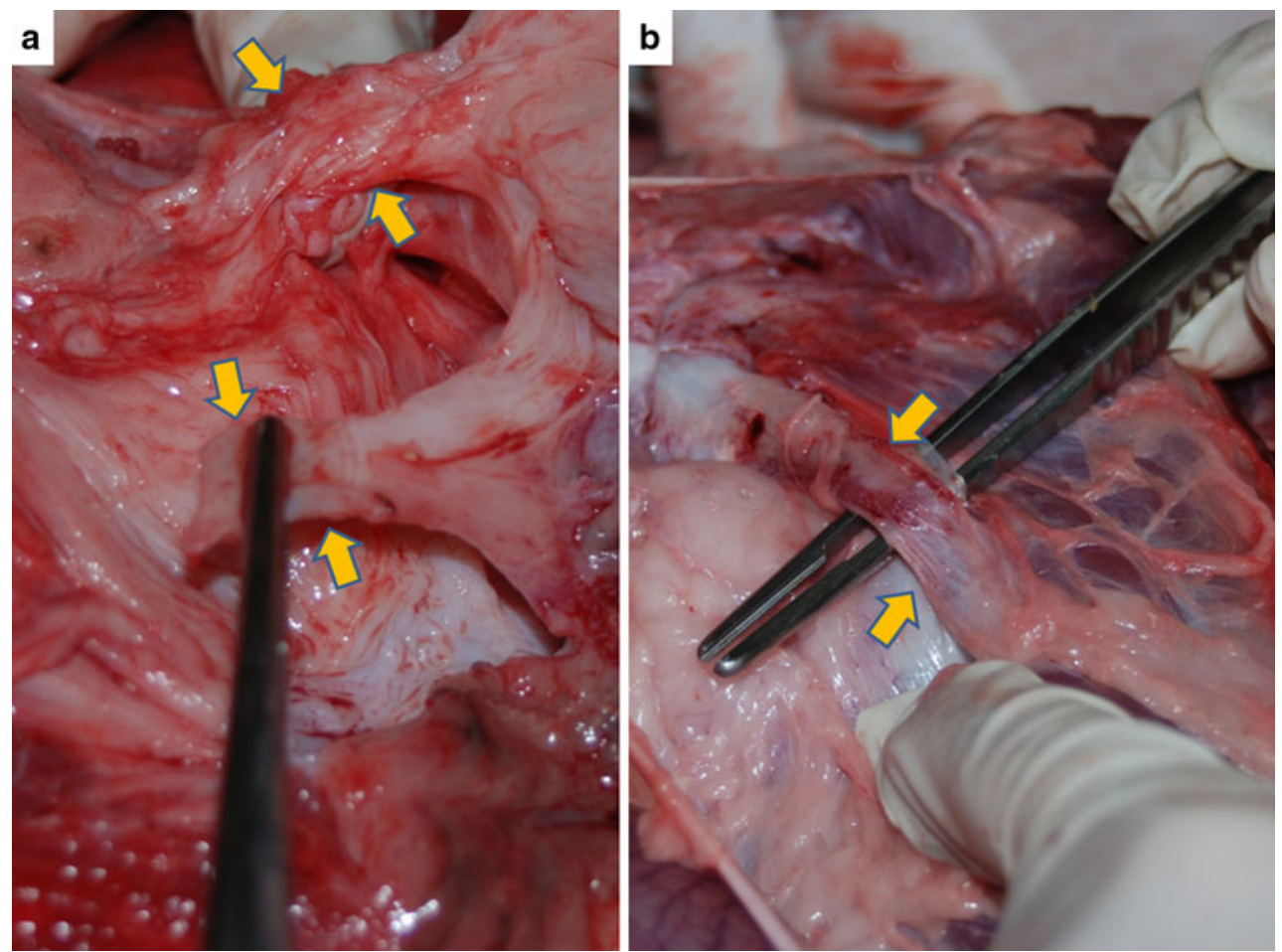

Fig. 4 a The straps 1 month after delivery: the straps are covered by thick fibrotic tissue, firmly integrated into the abdominal wall tissues. b The straps 4 months after delivery: complete incorporation of the arm within the surrounding tissue 


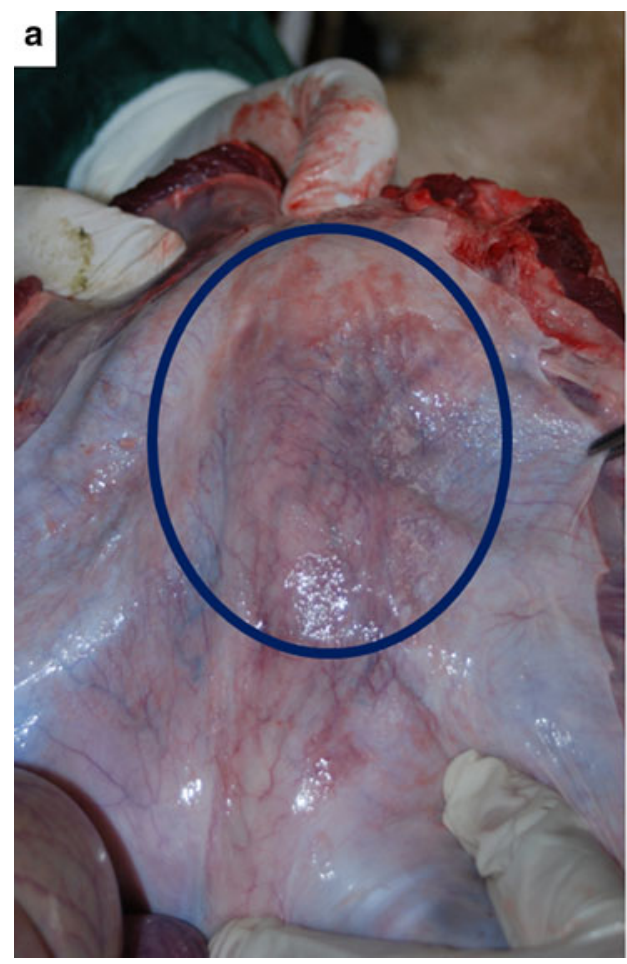

Fig. 5 The mesh 4 months after implantation. a Transperitoneal view of the mesh well deployed above the peritoneal sheath (blue circle). b The shape of the mesh after dissecting the peritoneum $(X)$. Of note,

\section{Discussion}

To date, in open ventral hernia surgery, one of the critical issues is related to the need for mesh fixation at its lateral margins to avoid mesh migration or dislocation due to physical movement. During ventral hernia procedures, the fixation of the mesh at the periphery of the abdominal wall has been shown to be challenging due to the anatomical limitations of space and access. For this reason, a minimally invasive procedure is often not possible and large wound openings are frequently required to facilitate suturing of the mesh in place. Besides an elevated frequency of postoperative bleeding, hematoma is reported to be a direct consequence of point fixation of the implant [14]. Due to the limitations of access and the need for multiple point fixation which tightly holds the mesh in place, we ask the question "Are mesh repairs always tension-free?" This often results in postoperative complications such as bleeding and hematoma. These complications are thought to be a result of point fixation tearing out of the tissue during stress loads on the implant postoperatively [14]. Moreover, tight suturing of the mesh to the muscular structures does not allow for a compliant motion of the implant, which may be helpful during movement of the abdominal wall. Eliminating the need for complex, multiple (often up to 20) point fixations should also simplify the

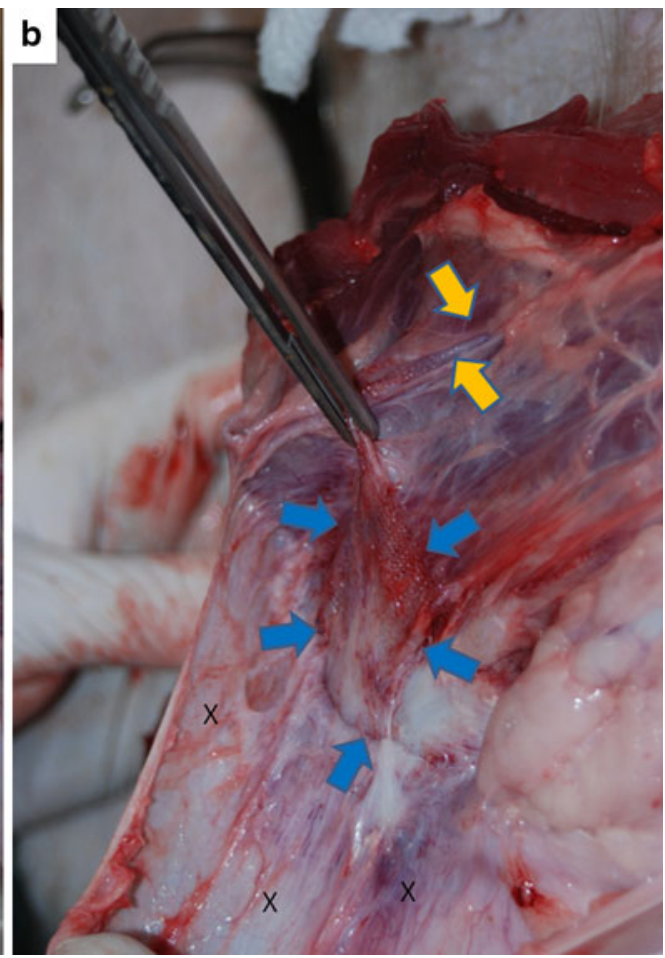

the flat and well-deployed contour of its preperitoneal surface (blue arrows). A strap looks firmly integrated in the abdominal wall tissue (yellow arrow)

surgical procedure, saving time, and could allow the reduction of the risks associated with those multiple fixations. The depicted arm system of our device is continuous with the body of the implant, and is made from the same mesh, which has give in the longitudinal direction. This allows the straps to extend under strain and motion, acting as a type of shock absorber. By distributing the strain of the implant along its flexible axis, the chance of disruption is reduced through the distribution of load, as reported in the literature [17].

In ventral hernia repair, the strap system could also allow for a much smaller wound opening by eliminating the need for complex suturing maneuvers. The simplified tunneling technique can be performed almost blind, and requires little to no supination and pronation within the confined space of the abdomen. In our experience, the arms appeared to have assured that the mesh remained correctly orientated in the abdominal wall and support our thoughts that the straps are functionally for orientation rather than fixation. Fixation occurs through tissue incorporation in the open weave of the mesh. This, we think, is crucial during the early postoperative period. The gripping forces exerted by the straps in the tissue tunnels is thought to be the consequence of physical compression, friction, and, soon after implantation, biological factors such as fibrin formation. The use of such polypropylene straps has been well 
established in pelvic organ prolapse repair. These tools have been demonstrated as very useful to achieve a fixation-free but firm and secure placement of polypropylene implants in high-pressure areas such as the pelvis [16-20]. The histological evidence of early fibroblast incorporation among the polypropylene straps, even after very short periods of time, is clearly demonstrated [20]. These features correspond to the histological findings that we have seen in the excised straps. Moreover, the histological results of the body of the mesh are in line with that described in the literature [13, 21-23].

Furthermore, the pullout force for the straps over varying time periods has been well evaluated in the literature. In fact, using straps with the same dimensions of the arms used in our study and in a comparable muscular structure, the force needed to remove the straps in human cadavers amounts to $1,176 \mathrm{~g}$ [24]. In another study concerning the features of the same device in animal models, it was demonstrated that the strap begins to incorporate within the tissues after just a few minutes. This last report showed that, immediately after placement, each strap required circa $282 \mathrm{~g}$ of pullout force to be removed but, due to full tissue incorporation, after 2 weeks, $4,457 \mathrm{~g}$ of pullout force were required to disrupt the mesh-this is well beyond the actual destruction of the tissues [25]. This type of stabilizing effect motivated us to describe this fixation-free arm system for ventral hernia repair with the neologism 'freexation.' The features of the 'freexated' arms illustrated in the present report could be significant, especially when considering the cascade of events typical of the early postoperative period. In this critical phase, in the case of strains or coughs, the compliant weave of the mesh will elongate, so that it does not permit abdominal forces to pull against fixed points, which would otherwise tear any fixation. In our opinion, this could be essential for the reduction of early recurrence due to implant dislodgement while the mesh is being integrated into the tissues. As there is such a wealth of literature showing the use of straps for mesh fixation in urogynecological procedures (preclinical and clinical), we deemed it only necessary to replicate the results as confirmation in an experimental trial in the porcine model. However, based upon the background knowledge and our own results, we consider the outcomes of this feasibility study very encouraging for the use of such a system in ventral hernia repair. Based upon this data, we believe that its use for ventral hernia repair in humans could shorten and simplify the procedure at the same time, reducing complications and recurrence rates through a broader, truly tension-free placement, and reduction of early disruption. Nevertheless, this will require comparative clinical trials for confirmation.

\section{References}

1. Junge $\mathrm{K}$, Klinge U, Prescher A, Giboni $\mathrm{P}$, Niewiera $\mathrm{M}$, Schumpelick V (2001) Elasticity of the anterior abdominal wall and impact for reparation of incisional hernias using mesh implants. Hernia 5:113-118

2. Bauer JJ, Harris MT, Gorfine SR, Kreel I (2002) Rives-Stoppa procedure for repair of large incisional hernias: experience with 57 patients. Hernia 6:120-123

3. Voeller GR, Ramshaw B, Park AE, Heniford BT (1999) Incisional hernia. J Am Coll Surg 189(6):635-637

4. White TJ, Santos MC, Thompson JS (1998) Factors affecting wound complications in repair of ventral hernias. Am Surg 64(3):276-280

5. Klinge U, Conze J, Klosterhalfen B, Limberg W, Obolenski B, Ottinger AP, Schumpelick V (1996) Changes in abdominal wall mechanics after mesh implantation. Experimental changes in mesh stability. Langenbecks Arch Chir 381(6):323-332

6. van der Linden FT, van Vroonhoven TJ (1988) Long-term results after surgical correction of incisional hernia. Neth J Surg 40(5):127-129

7. Langer S, Christiansen J (1985) Long-term results after incisional hernia repair. Acta Chir Scand 151:217-219

8. Geçim IE, Koçak S, Ersoz S, Bumin C, Aribal D (1996) Recurrence after incisional hernia repair: results and risk factors. Surg Today 26:607-609

9. McLanahan D, King LT, Weems C, Novotney M, Gibson K (1997) Retrorectus prosthetic mesh repair of midline abdominal hernia. Am J Surg 173(5):445-449

10. Arnaud JP, Tuech JJ, Pessaux P, Hadchity Y (1999) Surgical treatment of postoperative incisional hernias by intraperitoneal insertion of Dacron mesh and an aponeurotic graft: a report on 250 cases. Arch Surg 134:1260-1262

11. Langer C, Neufang T, Kley C, Liersch T, Becker H (2001) Central mesh recurrence after incisional hernia repair with Marlex-are the meshes strong enough? Hernia 5(3):164-167

12. Bauer JJ, Harris MT, Kreel I, Gelernt IM (1999) Twelve-year experience with expanded polytetrafluoroethylene in the repair of abdominal wall defects. Mt Sinai J Med 66:20-25

13. Amid PK, Shulman AG, Lichtenstein IL, Hakakha M (1994) Biomaterials for abdominal wall hernia surgery and principles of their applications. Langenbecks Arch Chir 379(3):168-171

14. Welty G, Klinge U, Klosterhalfen B, Kasperk R, Schumpelick V (2001) Functional impairment and complaints following incisional hernia repair with different polypropylene meshes. Hernia 5(3): 142-147

15. Awad ZT, Puri V, LeBlanc K, Stoppa R, Fitzgibbons RJ Jr, Iqbal A, Filipi CJ (2005) Mechanisms of ventral hernia recurrence after mesh repair and a new proposed classification. J Am Coll Surg 201(1):132-140

16. Boukerrou M, Rubod C, Dedet B, Boodhum R, Nayama M, Cosson M (2008) Tissue resistance of the tension-free procedure: what about healing? Int Urogynecol J Pelvic Floor Dysfunct 19(3):397-400

17. Fatton B, Amblard J, Debodinance P, Cosson M, Jacquetin B (2007) Transvaginal repair of genital prolapse: preliminary results of a new tension-free vaginal mesh (Prolift technique)-a case series multicentric study. Int Urogynecol J Pelvic Floor Dysfunct 18:743-752

18. Altman D, Väyrynen T, Engh ME, Axelsen S, Falconer C; Nordic Transvaginal Mesh Group (2008) Short-term outcome after transvaginal mesh repair of pelvic organ prolapse. Int Urogynecol J Pelvic Floor Dysfunct 19(6):787-793

19. Abdel-Fattah M, Ramsay I; West of Scotland Study Group (2008) Retrospective multicentre study of the new minimally invasive 
mesh repair devices for pelvic organ prolapse. BJOG 115(1):2230

20. Boccon-Gibod L, Hermieu JF, Toublanc M, Delmas V, Ravery V (2004) International Continence Society (ICS) Congress 2004, Abstract 681

21. Hernia Trialists Collaboration EU (2000) Mesh compared with non-mesh methods of open groin hernia repair: systematic review of randomized controlled trials. Br J Surg 87:854-859

22. DeBord JR (1998) The historical development of prosthetics in hernia surgery. Surg Clin North Am 78:973-1006
23. Read RC (2003) Recent advances in the repair of groin herniation. Curr Probl Surg 40(1):13-79

24. Malik B, Lambaudie E, Collinet P, Dubois P, Cosson M (2007) Mechanical resistance of synthetic meshes for incontinence or prolapse surgery. Int Urogynecol J 18:183-187

25. Alcalay M, Livne M, Shidlovsky D, Hod E (2009) Pullout force of polypropylene mesh deployed by EndoFast Reliant fastener-a comparative study in a sheep model. International Continence Society (ICS) Congress 2009, Abstract 543 